ARTICLE

DOI: $10.1038 / s 41467-017-02523-y$

\title{
Structural basis for chitin acquisition by marine Vibrio species
}

\author{
Anuwat Aunkham ${ }^{1}$ Michael Zahn ${ }^{2}$, Anusha Kesireddy ${ }^{3}$, Karunakar Reddy Pothula $^{3}$, Albert Schulte $^{4}$,
} Arnaud Baslé ${ }^{2}$, Ulrich Kleinekathöfer (1) ${ }^{3}$, Wipa Suginta ${ }^{1,5}$ \& Bert van den Berg (1) ${ }^{2}$

Chitin, an insoluble polymer of $\mathrm{N}$-acetylglucosamine, is one of the most abundant biopolymers on Earth. By degrading chitin, chitinolytic bacteria such as Vibrio harveyi are critical for chitin recycling and maintenance of carbon and nitrogen cycles in the world's oceans. $A$ decisive step in chitin degradation is the uptake of chito-oligosaccharides by an outer membrane protein channel named chitoporin (ChiP). Here, we report X-ray crystal structures of ChiP from $V$. harveyi in the presence and absence of chito-oligosaccharides. Structures without bound sugar reveal a trimeric assembly with an unprecedented closing of the transport pore by the $\mathrm{N}$-terminus of a neighboring subunit. Substrate binding ejects the pore plug to open the transport channel. Together with molecular dynamics simulations, electrophysiology and in vitro transport assays our data provide an explanation for the exceptional affinity of ChiP for chito-oligosaccharides and point to an important role of the $\mathrm{N}$ terminal gate in substrate transport.

\footnotetext{
${ }^{1}$ Biochemistry-Electrochemistry Research Unit, Institute of Science, Suranaree University of Technology, Nakhon Ratchasima 30000, Thailand. ${ }^{2}$ Institute for Cell and Molecular Biosciences, The Medical School, Newcastle University, Newcastle upon Tyne NE2 4HH, UK. ${ }^{3}$ Department of Physics \& Earth Sciences, Jacobs University Bremen, 28759 Bremen, Germany. ${ }^{4}$ School of Biomolecular Science and Engineering, Vidyasirimedhi Institute of Science and Technology (VISTEC), Wangchan Valley, 555 Moo 1 Payupnai, Wangchan, Rayong 21210, Thailand. ${ }^{5}$ Center of Excellence in Advanced Functional Materials, Suranaree University of Technology, Nakhon Ratchasima 30000, Thailand. Anuwat Aunkham and Michael Zahn contributed equally to this work. Correspondence and requests for materials should be addressed to W.S. (email: wipa@sut.ac.th) or to B.v.d.B. (email: Bert.Van-Den-Berg@newcastle.ac.uk)
} 
C hitin, an insoluble polymer of $\beta 1,4$-linked $N$-acetylglucosamine (GlcNAc) residues, is one of the most abundant biopolymers on Earth, along with cellulose $e^{1,2}$. Chitin serves as the major structural component of arthropods, the cell walls of some fungi, and the primary exoskeletons of crustaceans and marine zooplankton ${ }^{3}$. The global estimation of chitin production is $\sim 10^{10}-10^{11}$ tons $^{4}$. Remarkably, while providing a constant rain of polysaccharides to the ocean floor ("marine snow"), no substantial accumulation of chitin in ocean sediments occurs due to the rapid recycling of chitin driven by chitinolytic bacteria, mainly from the family Vibrionaceae ${ }^{5,6}$. These bacteria utilize only chitin as a sole source of cellular energy, and certain Vibrio species such as Vibrio harveyi and Vibrio parahaemolyticus are extremely fast growing. Hence, these bacteria play critical roles in maintaining the carbon and nitrogen cycles in marine ecosystems.

The chitin utilization pathway of marine Vibrios is highly conserved and incorporates a number of characterized and uncharacterized enzymes, chitin binding proteins, and transport proteins $s^{7,8}$. Proteins involved in the initial processes include chemotaxis proteins responsive to chitin oligosaccharides and extracellular chitinases that degrade the insoluble polymer into water-soluble chitooligosaccharides that are imported across the outer membrane (OM) through a dedicated, chitooligosaccharide-specific channel ${ }^{9-11}$. In the periplasmic space, chitin dextrinase $\mathrm{e}^{12}$ and $\beta-N$-acetylglucosaminidase $\mathrm{e}^{13,14}$ generate the mono- (GlcNAc) and disaccharide $\left(\mathrm{GlcNAc}_{2}\right)$ that are subsequently transported into the cytoplasm via several $\mathrm{ABC}$ transporter complexes in the inner membrane. Finally, at least six cytoplasmic enzymes convert the transport products to fructose6-P, $\mathrm{NH}_{3}$, and acetate ${ }^{7}$.

Cloning of a number of chitin utilization genes has been reported from Vibrio furnissii, Vibrio cholera, and V. harveyi and the corresponding proteins have been characterized $9,10,12,15-20$. A key step in chitin utilization represents the cellular acquisition of soluble chitin oligosaccharides $\left(\mathrm{GlCNAc}_{2-6}\right)$ produced by the action of extracellular chitinases ${ }^{20}$. This uptake process is carried out by an OM diffusion channel, termed chitoporin (ChiP) ${ }^{9-11}$. ChiP was first identified in V. furnissii and was proposed to be a chitooligosaccharide-uptake channel based on expression profiles in the presence of various sugar substrates ${ }^{21}$. DNA microarray expression profiles further demonstrated that expression of the chiP gene in $V$. cholerae is induced by chitin oligosaccharides and that the genes responsible for chitin degradation are under the stringent control of the chiS regulon ${ }^{21}$. The gene encoding for ChiP is found in most marine members of the Vibrionaceae, emphasizing the general importance of this OM diffusion channel for chitin utilization.

On a molecular level, it is so far unclear how ChiP mediates the specific uptake of chitooligosaccharides. The protein from $V$. harveyi (denoted VhChiP) has recently been cloned and expressed at high levels in Escherichia coli and purified to homogeneity. VhChiP was purified as a stable trimer, suggesting a structural similarity to the general diffusion porins $\mathrm{OmpF}$ and $\mathrm{OmpC}$ from E. coli. In single-channel electrophysiology, VhChiP displays a trimeric conductance of $1.8 \mathrm{nS}$ in $1 \mathrm{M} \mathrm{KCl}$, which is half of the trimeric conductance of $E$. coli $\mathrm{OmpF}(4.2 \mathrm{nS})^{22}$ but substantially larger than its well-characterized sugar-specific analog LamB (maltoporin) from E. coli $(160 \mathrm{pS})$ and Salmonella typhimurium $(90 \mathrm{pS})^{23,24}$. The addition of micromolar concentrations of chitooligosaccharides such as chitohexaose $\left(\mathrm{GlCNAc}_{6}\right)$ causes a complete block of ionic current, demonstrating tight binding. Interestingly, closely related sugars such as maltohexaose do not interact with the channel, suggesting remarkable substrate specificity of $V h$ ChiP. The binding constant $(K)$ for the most potent substrate chitohexaose is about $5 \times 10^{6} \mathrm{M}^{-1}$, which is at least an
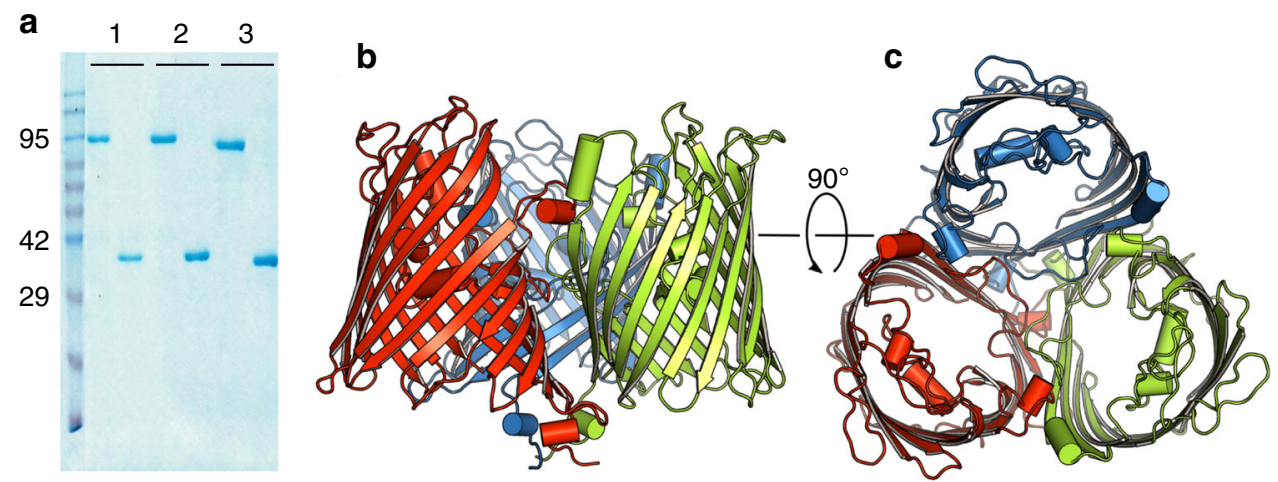

d
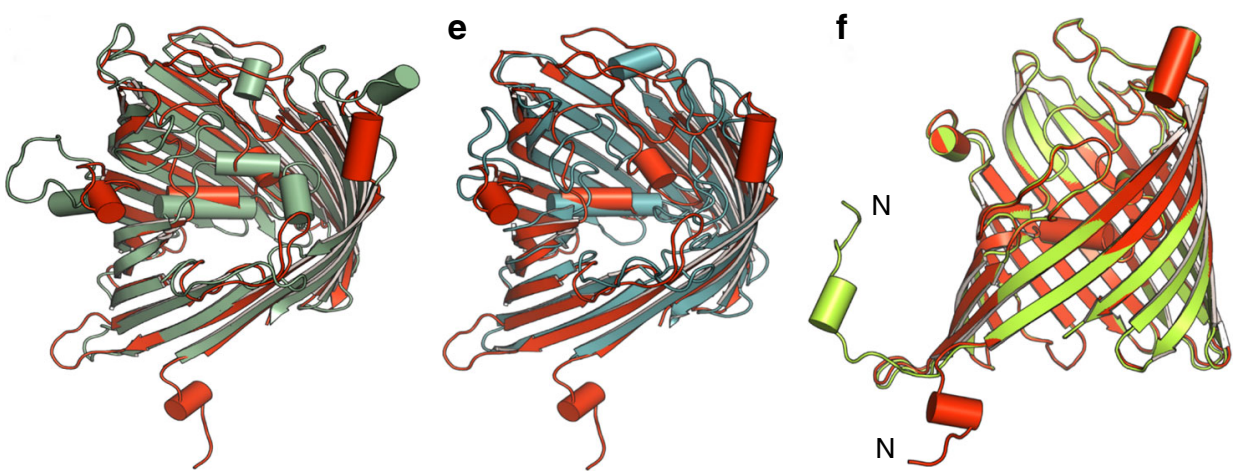

Fig. 1 X-ray crystal structure of in vitro-folded VhChiP. a SDS-PAGE gel of OM-expressed (native; 1), in vitro-folded (2) and truncated VhChiP (3) loaded both as non-boiled (left lanes) and boiled samples (right lanes). b Side view and $\mathbf{c}$ top view cartoon presentations of the in vitro-folded $\mathrm{VhChiP}$ trimer. $\mathbf{d}$, $\mathbf{e}$ Top view superpositions of in vitro-folded VhChiP (red) with Neisseria meningitidis PorB (green; $\mathbf{d}$ ) and Delftia acidovorans Omp32 (blue; e). f Superposition of in vitro-folded VhChiP (red) with OM-expressed VhChiP (green). The N-termini have been labeled 
order of magnitude higher compared to other OM sugar-specific diffusion channels ${ }^{11}$. In vitro liposome swelling assays confirm the highly specific nature of the channel for chitooligosaccharides $^{10}$. Statistical analysis on stochastic fluctuations of ion current through VhChiP in the presence of chitohexaose indicated that $V h$ ChiP has multiple binding sites for sugar, and the trapping properties of chitoporin exhibit memory effects, given that the average binding rate of an unblocked monomer is larger when its neighboring monomers are blocked. Such results suggested a possible design strategy to enhance the rate of sugar uptake by the bacterium $^{25,26}$. A homology model based on the X-ray crystal structure of Delftia acidovorans Omp32 (pdb ID: 2FGR $)^{27}$ predicted Trp136, located at the mouth of the channel constriction, to be an important residue for chitin transport ${ }^{28}$. This notion was confirmed by single-channel electrophysiology and liposome swelling experiments, showing that for the preferred substrate chitohexaose, mutation of Trp136 led to decreased binding affinity and reduced rates of uptake respectively.

Here, we report X-ray crystal structures of VhChiP in the absence and presence of chitooligosaccharides. Together with single-channel electrophysiology and molecular dynamics (MD) simulations the results reveal the structural basis for the exquisite substrate specificity of chitoporin and clarify the mechanism of facilitated diffusion of chitooligosaccharides across the OM of Vibrionaceae.

\section{Results}

Purification and crystallization of VhChiP. We expressed VhChiP without a His-tag in the OM of the porin-deficient E. coli
Bl21 omp8 strain and obtained reasonable yields ( $3 \mathrm{mg}$ from 121 of culture) even though $V h$ ChiP overexpression appeared to be highly toxic for E. coli. The protein was expressed without a tag based on the predicted similarity to general porins in which the $\mathrm{N}$ - and C-termini of the monomers interact to form a salt bridge. Figure 1a shows that boiled and non-boiled SDS-PAGE samples have different mobilities, with trimers observed in non-boiled samples and monomers after boiling, i.e., VhChiP is heatmodifiable. The purified protein was crystallized in the presence of $0.4 \% \mathrm{C}_{8} \mathrm{E}_{4}$ as detergent (Methods). The obtained crystals of VhChiP in space group C2 diffracted to reasonable resolutions of $\sim 2.5 \AA$ (Supplementary Table 1). Despite this, structure solution using molecular replacement (MR) failed, presumably due to the lack of a good homology model given that sequence identities of likely search models with VhChiP are $20 \%$ or less. Soaking of native crystals with different heavy atoms was also not successful. Next, we attempted to express VhChiP in minimal media in the presence of seleno-methionine (SeMet) for phasing using single/ multiple anomalous dispersion (SAD/MAD) approaches, but the cells failed to grow in accordance with the protein toxicity that was observed in rich medium. Subsequently we cloned VhChiP without a signal sequence and with a C-terminal $\mathrm{His}_{6}$-tag into pET28a (Methods) to express the protein into inclusion bodies (IBs) and avoid toxicity issues. As expected, the cells grew now very well in minimum medium in the presence of SeMet. VhChiP from IBs was folded in vitro from $8 \mathrm{M}$ urea and purified as the native protein (Methods). Well-diffracting crystals $(\sim 2 \AA$ resolution) in space group $\mathrm{P} 21$ were obtained and a SAD data set was
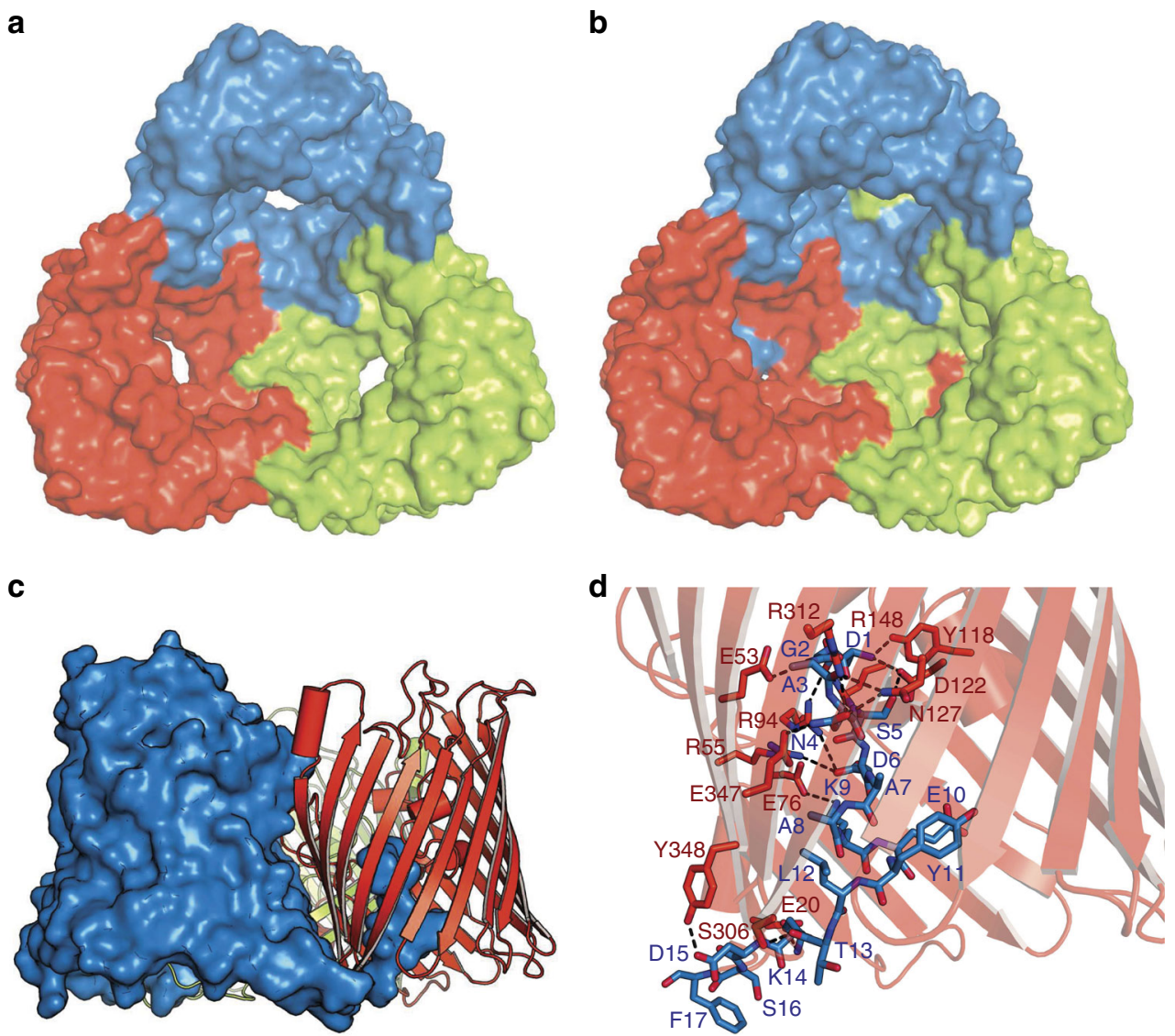

Fig. 2 OM-expressed VhChip has N-terminally plugged channels. a, b Surface representations (top view) of in vitro-folded VhChiP (a) and natively expressed $V h C h i P(\mathbf{b})$. The barrel lumen of the natively expressed $V h C h i P$ is occupied by the $\mathrm{N}$-terminus of a neighbor barrel. $\mathbf{c} \mathrm{N}$-terminal insertion mode of natively expressed VhChiP. The N-terminus of one barrel (blue) plugs the lumen of a neighbor barrel (red). $\mathbf{d}$ Polar interactions between the inner barrel wall (red) with the $\mathrm{N}$-terminus of a neighbor barrel (blue) 
collected at the absorption edge of Selenium. The structure was solved using Autosol in Phenix and refined in Refmac (Methods).

VhChiP forms trimers with $\mathrm{N}$-terminally plugged pores. In vitro-folded VhChiP forms a trimeric assembly of oval $\beta$-barrels, each consisting of 16 strands (Fig. 1), like the general diffusion porins OmpF/C from E. coli. Unlike OmpF/C however, the $\mathrm{N}$ terminus of VhChiP does not form an intramolecular salt bridge with the C-terminal carboxyl group. Instead, the first $\mathrm{N}$-terminal 17 amino acids of VhChiP are not part of the barrel and extend into the periplasm where they form a trimerization motif like that observed in the phosphate transporter OprP from Pseudomonas aeruginos $a^{29}$. The first nine amino acids of the $\mathrm{N}$-terminus are disordered and not visible in the electron density (Fig. 1b). The overall topology of the barrel, including the periplasmic $\mathrm{N}$ terminal region, is correctly predicted by Boctopus and PRED$\mathrm{TMBB}^{30,31}$. A DALI search ${ }^{32}$ identifies $N$. meningitidis PorB, which has $18 \%$ sequence identity to VhChiP, as the closest structural homolog in the database $(Z=29$; r.m.s.d. $2.7 \AA$ over 301 residues). The second-highest similarity is observed for Omp32 from $D$. acidovorans $(Z=26$; r.m.s.d. $2.7 \AA$ over 295 residues), which was used for previous homology modeling of VhChiP. Overall, the differences between both structural homologs and VhChiP are substantial, especially for most extracellular loops and the functionally important, barrel constricting loop L3 (Fig. 1 d, e).

The crystal structure of the OM-expressed channel is almost identical to that of the in vitro-folded protein. Strikingly however, in OM-expressed VhChiP the N-termini plug the pore of a neighboring $\beta$-barrel within the trimeric assembly, effectively blocking all $\beta$-barrels for substrate transport (Fig. 2). The blocking of a channel by a neighbor within an oligomeric assembly is, to our knowledge, unprecedented. The reason that the plug is inter- and not intra-molecular might be due to fact that the $\mathrm{N}$-terminal $\beta$-strand is pointing away from its own pore towards the neighboring one (Fig. If and Supplementary Fig. 1). This is due to the small angle of the strand(s) relative to the OM plane. Thus, to plug its own pore, the N-terminus would have to fold back on itself in a rather elaborate way and would likely need to be longer. All residues of the $\mathrm{N}$-terminus have good electron density in the structure (Supplementary Fig. 1) and a number of polar interactions can be identified between the $\mathrm{N}$-terminal plug and the barrel, most notably between Asp1 (carboxylate)-Arg94/ 148 , Asp1 ( $\alpha$-amino group)-Y118/Asp122, Ala3-Glu53, Asn4Asn127/Arg312/Glu347, Ser5-Asp122, Asp6-Arg94, and Lys9Glu76 (Fig. 2). Thus, five out of the first six residues of the Nterminus make polar interactions with the pore of the protein, suggesting that the plug is stably inserted in the pore. It should be noted that for in vitro-folded VhChiP, two residues (MG) were added to the $\mathrm{N}$-terminus of the mature protein for inclusion body expression, and it is possible that the presence of those additional residues has destabilized the plug sufficiently for it be outside the pore.

To explore the stability of the pore plug further, we performed $500 \mathrm{~ns}$ equilibrium MD simulations of in vitro-folded and natively expressed $V h \mathrm{ChiP}$, with free periplasmic and poreinserted $\mathrm{N}$-termini respectively. The $\mathrm{N}$-terminal ten residues of in vitro-folded VhChiP are highly flexible and do not insert into the pore (Fig. 3). As expected from the interactions observed in the crystal structure, the plug in natively expressed $V h C h i P$ is very stable with root mean square fluctuations (RMSF) of about $1.5 \AA$. Interestingly, in the presence of the pore plug, residues 143-146 located in loop L3 show enlarged RMSF values (Fig. 3a). The movements in loop L3 are likely to be induced by fluctuations of the tail of the plug within the pore, and we hypothesize that these
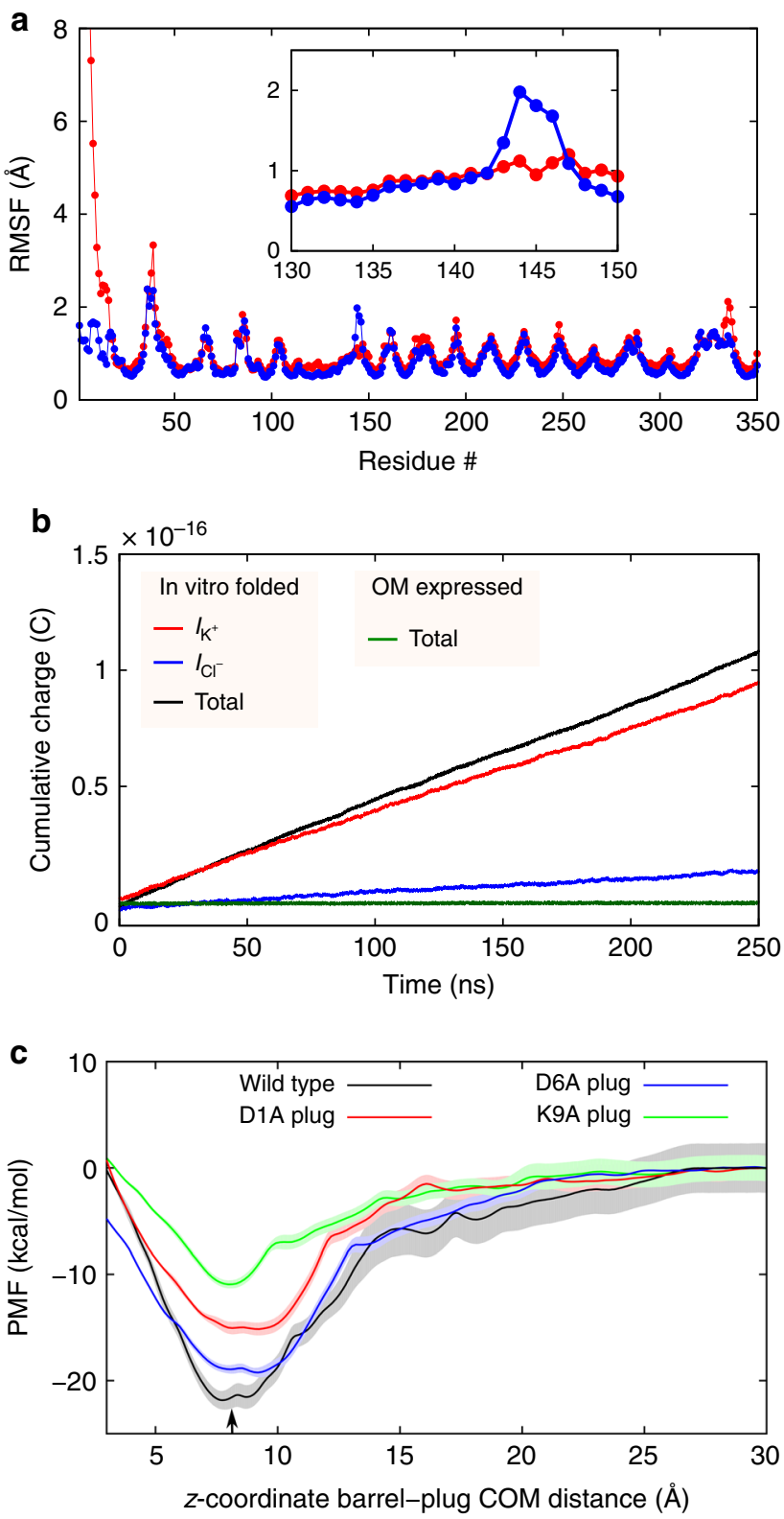

Fig. 3 Behavior of the pore plug in MD simulations. a Average $\mathrm{C} \alpha$ RMSF of the protein dynamics in vitro-folded (red) and OM-expressed (blue) VhChiP. In addition to the differences for the first 14 residues, a difference in the RMSF for residues 143-146 in loop L3 can be clearly observed. b Cumulative total charge as a function of time for a $1 \mathrm{M} \mathrm{KCl}$ solution at 200 $\mathrm{mV}$. In addition, the cumulative charges for $\mathrm{K}^{+}$and $\mathrm{Cl}^{-}$ions separately are shown as well. The data was averaged over the three simulations and the slopes of these curves yield the respective currents. c Free-energy surfaces of unbinding of the $\mathrm{N}$-terminus in wild type and mutant channels. The shaded areas indicate the respective error estimates. The arrow represents the position of the $\mathrm{N}$-terminal plug in the crystal structure of OM-expressed VhChip

concerted motions might initiate the N-terminal unbinding process that has to occur in order for substrate to bind.

In a next step, $\mathrm{MD}$ simulations $(3 \times 250 \mathrm{~ns})$ were performed to estimate the conductance of the in vitro-folded channel in a $1 \mathrm{M}$ $\mathrm{KCl}$ solution in the presence of a $200 \mathrm{mV}$ membrane potential. Consistent with the experiments, the in vitro-folded trimeric channel shows a theoretical conductance value of $2.13 \pm 0.15 \mathrm{nS}$, only slightly higher than the experimental value of $1.8 \mathrm{nS}$ 
a

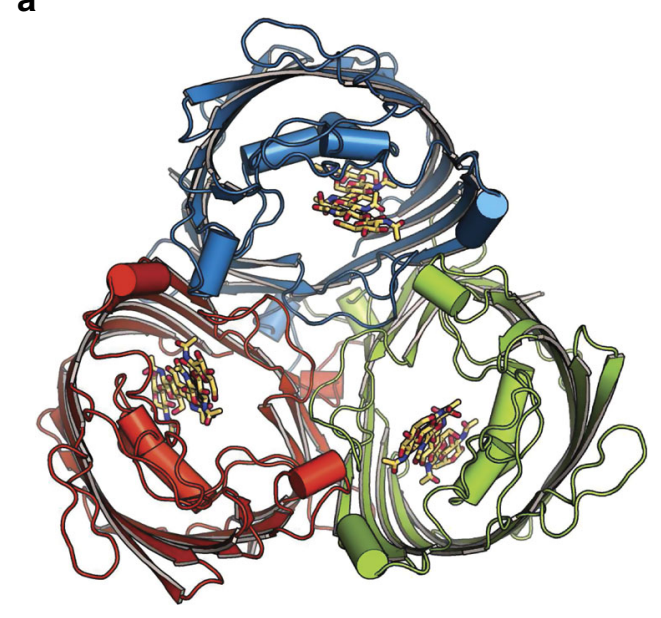

b

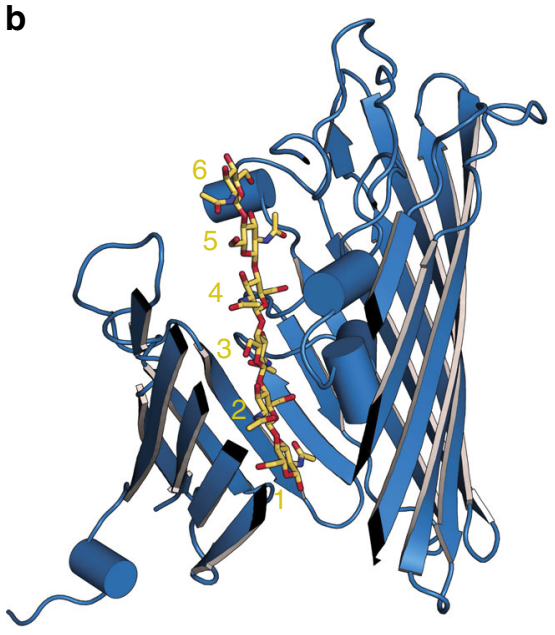

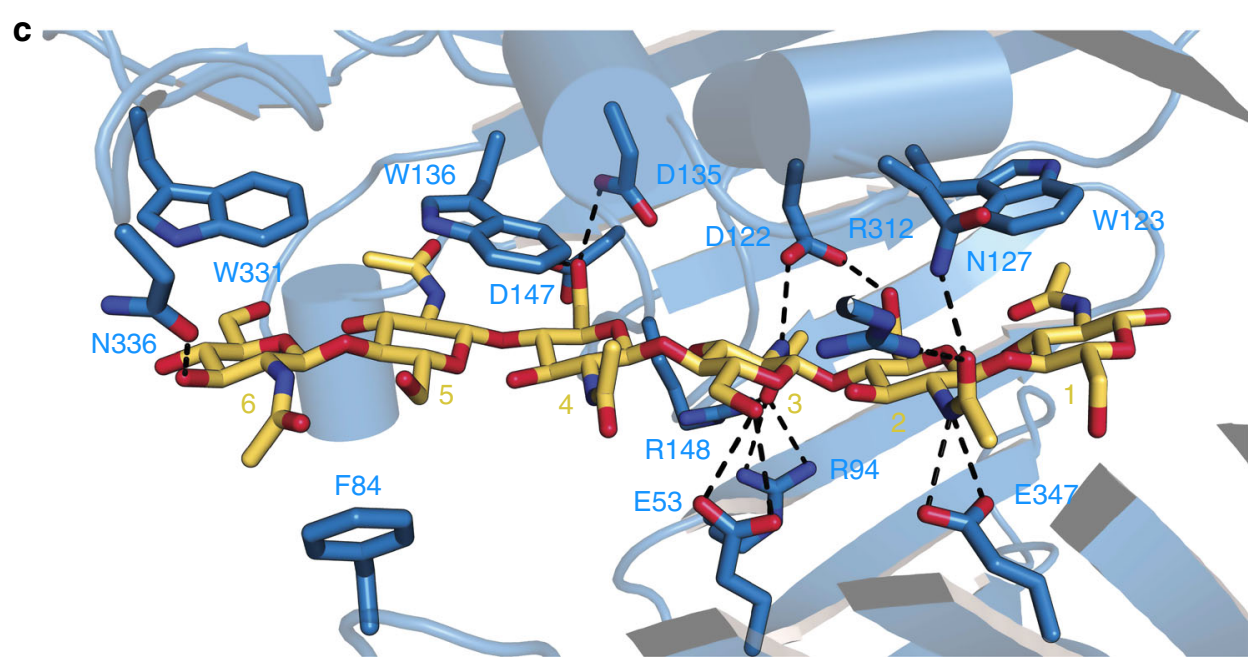

Fig. 4 Chitohexaose binding to in vitro-folded VhChiP. a Overview from the extracellular side. b Slabbed view from the side, with substrate residues labeled. c Schematic, showing interactions of key VhChiP residues with chitohexaose

measured at lower voltages ${ }^{22}$. When the plug is inside the pore, only very few ions can pass the plug and no statistically meaningful current value can be determined in the simulation time (Fig. 3b).

To obtain a more quantitative result concerning the binding energies of the N-termini, we determined one-dimensional freeenergy surfaces for wild type and D1A, D6A and K9A N-terminal plug mutants (Methods) using the umbrella sampling technique with two $2.24 \mu$ s long simulations. The mutated residues are involved in electrostatic interactions with channel-lining residues (Figs 2 and 3) and are therefore likely to be important for the stability of the plug-inserted state. As can be seen in Fig. 3c, the binding energy of the wild type $\mathrm{N}$-terminus is about $22 \mathrm{kcal} \mathrm{mol}^{-1}$ and the profile shares similar features as that obtained recently for the monomeric cyclodextrin channel $\mathrm{CymA}^{33}$. The D6A and D1A mutants had relatively little effect, while the K9A mutant reduced the binding energy roughly by a factor of two (Fig. 3c). The result suggests that Lys9 plays an important role in the stability of the plug-inserted state. Intriguingly, in CymA it has been verified experimentally that a similar positive residue is involved in the stability of the plug-bound conformation ${ }^{33}$. It is important to note that all determined free-energy surfaces show only one minimum, which is for the $\mathrm{N}$-terminus located inside the pore. This strongly supports the stability of the closed state of the VhChiP channel as observed in the crystal structure of the natively expressed protein.
Chitohexaose binding to in vitro-folded VhChiP. To obtain a structure of VhChiP in complex with a substrate, we cocrystallized in vitro-folded VhChiP with the substrate chitohexaose. Well-diffracting crystals grew under the same conditions and in the same space group as for the apo protein (Supplementary Table 1). Following MR, density was observed for all six units of the $\mathrm{GlcNAc}_{6}$ oligosaccharide (Fig. 4 and Supplementary Fig. 2). The substrate is bound in an extended conformation, with the density for the GlcNac units at both ends of the substrate (GlcNac-1 and -6) somewhat less clear compared to that for the central GlcNac-2-GlcNac-5 units. However, the density is of sufficient quality for placement of the substrate molecule with its reducing end (denoted GlcNAc-1) on the periplasmic side (Supplementary Fig. 2), stacked against the aromatic ring of Trp123. On the extracellular side of the channel constriction, the last sugar unit (denoted GlcNAc-6) stacks against Trp331 (Fig. 4). The same orientation of substrate (reducing end periplasmic) has been observed for maltoporin, albeit at lower resolution $(2.8 \AA)^{34}$.

Besides the ring stacking, few other interactions are present between GlcNAc-1 and GlcNAc- 6 and VhChiP. The aromatic ring of Trp136 stacks against both GlcNAc-4 and GlcNAc-5, providing a clear explanation as to why this residue is important for chitohexaose binding by $V h \mathrm{ChiP}^{28}$. Both Trp123 and Trp136 are conserved in VhChiP orthologs, whereas Trp331 is not (Fig. 5). The structure provides a clear rationale for the fact that $\left(\mathrm{GlcNAc}_{6}\right)$ is the best substrate for VhChiP, since beyond 


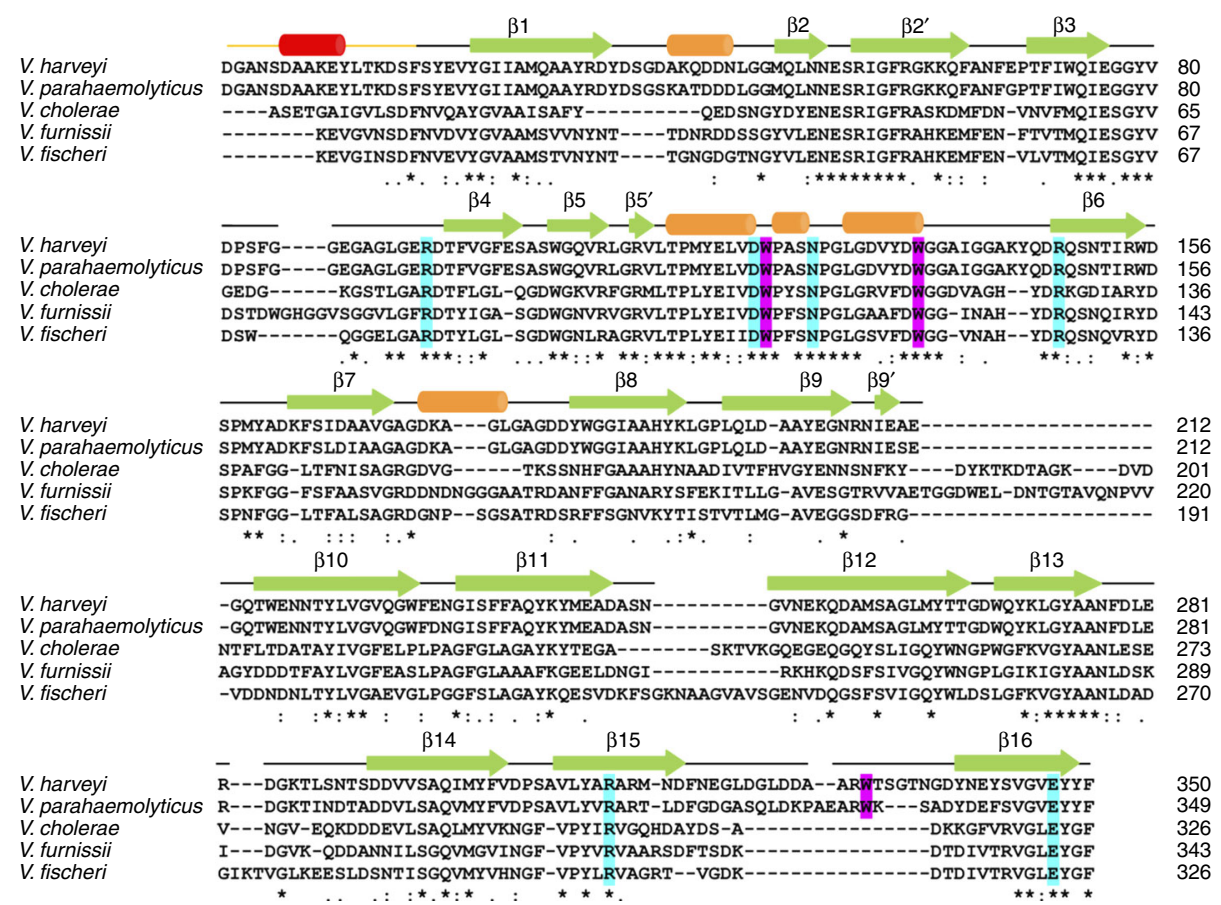

Fig. 5 Alignment of VhChiP orthologs from Vibrio species. Observed secondary structure elements have been indicated (orange cylinders, helices; green arrows, $\beta$-sheets). The first helix that is part of the $\mathrm{N}$-terminal plug is presented in red. Key aromatic (purple) and hydrophilic residues (cyan) interacting with chitooligosaccharides are colored. The following orthologs have been aligned: V. harveii (GenBank ID: HF558985.1), V. parahaemolyticus (GenBank ID: CP012950.1), V. cholera (GenBank ID: DQ774012.1), V. furnissii (GenBank ID: AF129934.1), and V. fischeri (GenBank ID: CP001139.1). Chitoporin from V. harveyi (UniProtKB/TrEMBL entry: LORVUO) was used as protein template to identify putative chitoporins. The alignment was generated using "CLUSTALW" algorithm in the DNASTAR package and displayed in Genedoc. The secondary structure of VhChiP was generated by ESPript v. 2.2

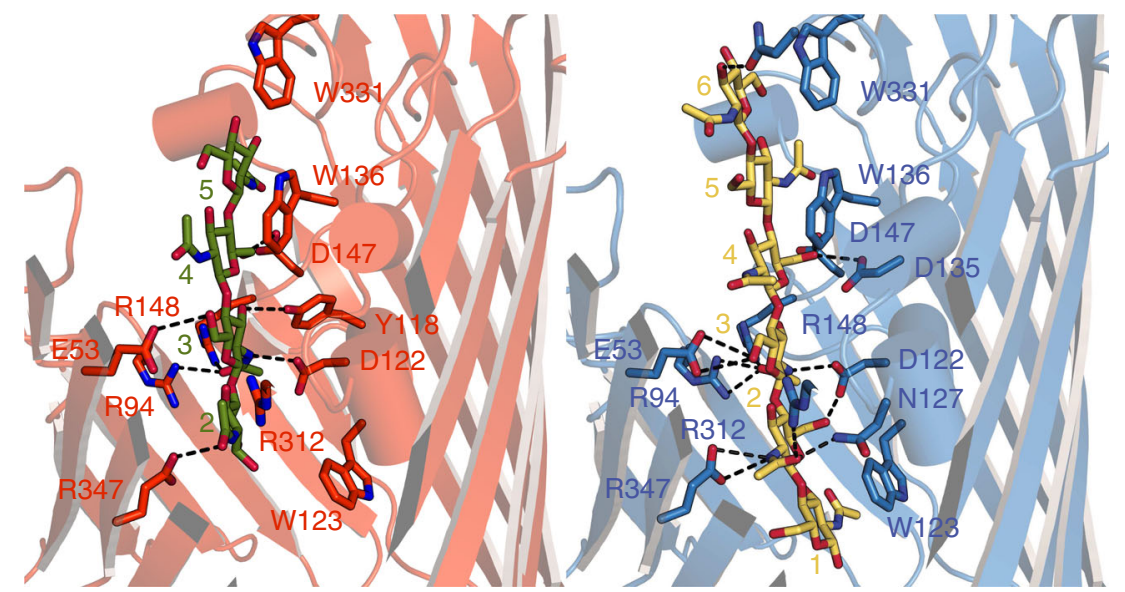

Fig. 6 Chitotetraose binding to VhChiP displaces the N-terminal plug. Side views in the same orientations, showing the bound chitotetraose in natively expressed VhChiP (left panel) and chitohexaose in in vitro-folded VhChiP (right panel). Residues interacting with the sugars are labeled

GlcNAc-1 and GlcNAc-6 the channel widens, decreasing the potential for interactions between the substrate and the channel. The central four residues of the sugar chain (GlcNAc-2 to GlcNAc-5) form several polar interactions with the channel interior, some of which are mediated by water molecules (Fig. 4). Importantly, the density for the central part of GlcNAc $\mathrm{C}_{6}$ is welldefined and allows unambiguous assignment of the acetamido groups, which for successive GlcNAc units point in opposite directions, as also observed for the chitohexaose molecule bound to the E. coli transglycosidase MltA ${ }^{35}$. The acetamido carbonyl groups of GlcNAc-2 and GlcNAc-3 are likely to be especially important for binding. The former interacts closely with the amide of Asn127 and to a lesser extent with Arg312, whereas the latter makes strong hydrogen bonds to the side chains of Arg94 and Arg148 (Fig. 4). In addition, the acetamido amides of GlcNAc-2 and GlcNAc-3 interact with the carboxylates of Glu347 and Asp122 respectively.

Chitotetraose binding to natively expressed $\mathbf{V h C h i P}$. Given that the channels of the OM-expressed apo-protein are closed due to the insertion of the N-terminus of a neighboring monomer (Fig. 2), it is important to establish whether the incoming substrate from the extracellular space could displace the $\mathrm{N}$-terminus to bind in the pore. In addition, we wanted to establish how chitooligosaccharides of different lengths bind to VhChiP. To 

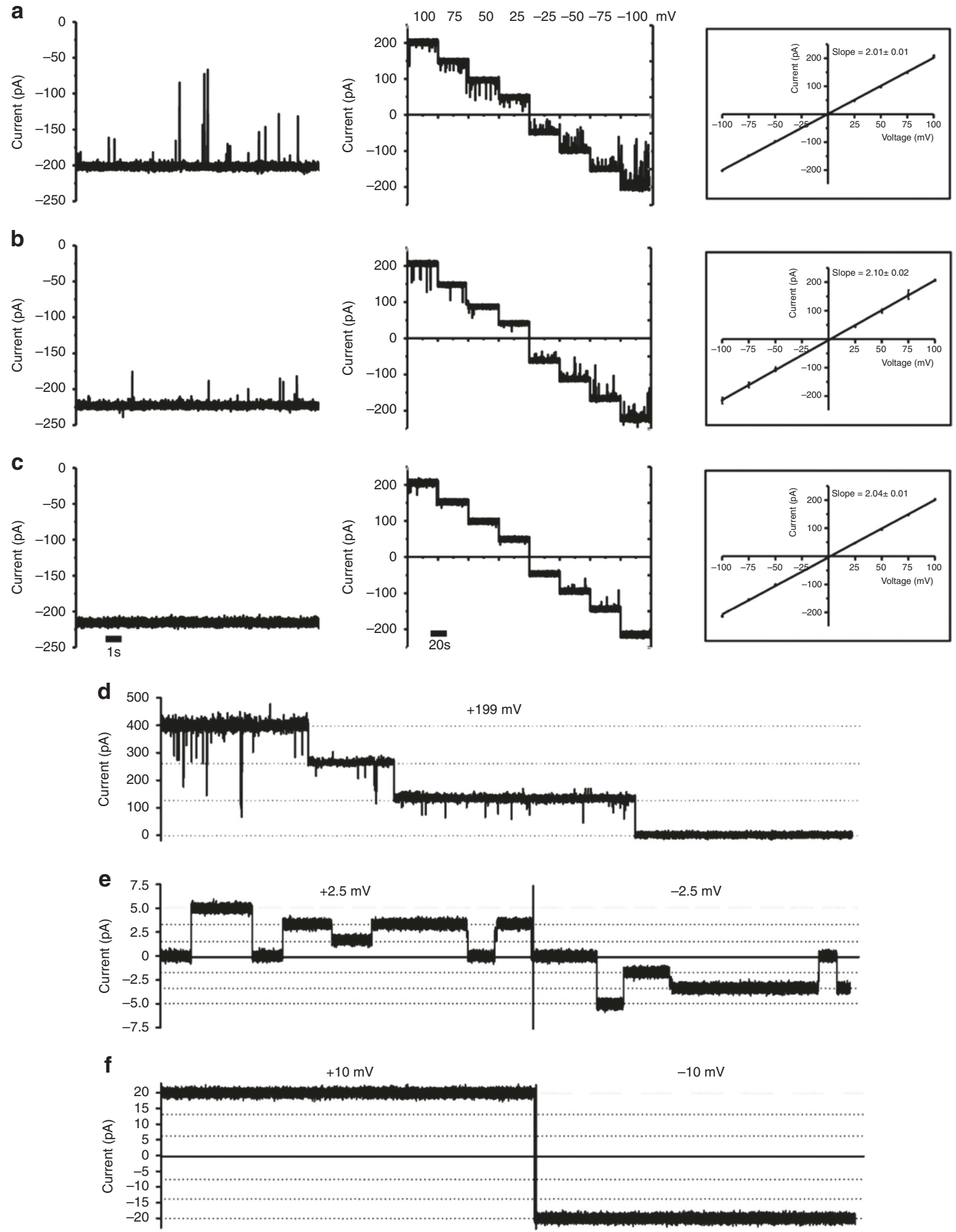

Fig. 7 Voltage-induced ejection of the pore plug in VhChiP. a-c Single-channel electrophysiology of OM-folded VhChiP (a), in vitro-folded VhChiP (b) and truncated $\mathrm{VhChiP}(\mathbf{c})$. Left panels show typical traces at $-100 \mathrm{mV}$, whereas the center and right panels show the current-voltage profiles. Data represent mean \pm s.d., $n=3$. d Stepwise voltage-induced closure of OM-folded VhChiP channels at $200 \mathrm{mV}$. e, $\mathbf{f}$ Typical traces observed at $\pm 2.5 \mathrm{mV}(\mathbf{e})$ and at \pm 10 $\mathrm{mV}(\mathbf{f})$. The data shown in $\mathbf{d}-\mathbf{f}$ are for the same experiment, with protein only added at the start (d). Traces shown are representative of three independent experiments

answer these questions we co-crystallized OM-expressed VhChiP with chitotetraose $\left(\mathrm{GlcNAc}_{4}\right)$. OM-expressed VhChiP in the presence of the $\mathrm{GlcNAc}_{4}$ substrate also crystallizes under the same conditions and in the same space group as the closed apoprotein (Supplementary Table 1). Importantly, the oligosaccharide is bound in the channel and has displaced the N-terminus, which is now in the periplasmic space and has the same conformation as that of the in vitro-folded protein.

The $\mathrm{GlNAAc}_{4}$ substrate occupies approximately the positions of GlcNAc-2 to GlcNAc-5 of the GlcNAc 6 chain (Fig. 6), suggesting that the central positions of the binding site provide the most binding energy for chitooligosaccharides (note that the subunits 
a

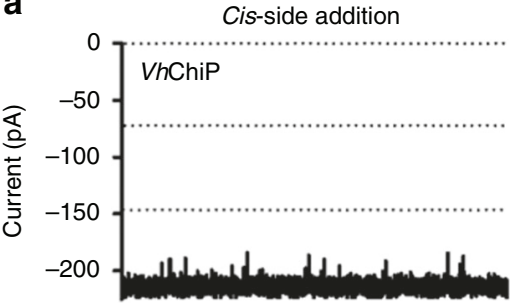

b

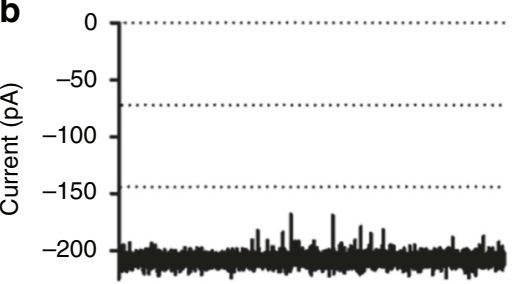

C

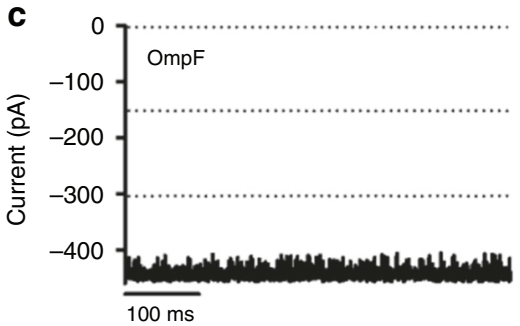

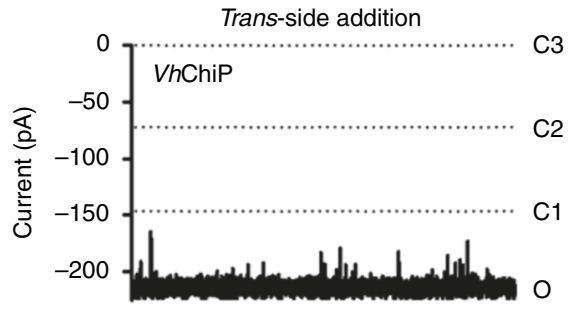
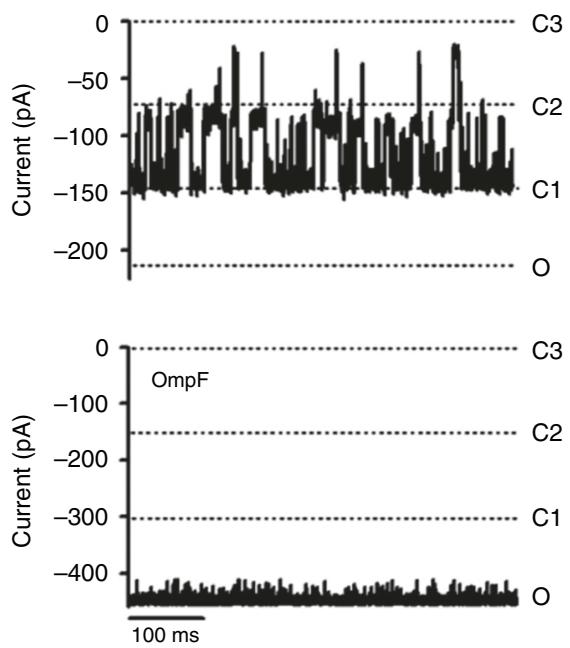

Fig. 8 Channel blockage by an N-terminal plug peptide. Single-channel current traces at $-100 \mathrm{mV}(1 \mathrm{M} \mathrm{KCl})$ of truncated VhChiP in the absence (a) and presence of $100 \mu \mathrm{M}(\mathbf{b})$ of the plug peptide DGANSDAAK. c E. coli OmpF traces in the presence of $200 \mu \mathrm{M}$ peptide. Traces shown are representative of three independent experiments

of $\mathrm{GlCNAc}_{4}$ are numbered 2-5 to provide consistency with those of chitohexaose). However, there are differences of up to $5 \AA$ for individual atoms between the two structures, especially at both ends of the substrate (GlcNAc-2 and GlcNAc-5). In most cases, however, this does not dramatically change the interactions between the sugar and the channel. For example, the acetamido carbonyl group GlcNAc-2 interacts in both structures with Asn127 and Arg312 whereas that of GlcNAc-3 interacts with Arg94/148 (Fig. 6). It appears therefore that VhChiP accommodates compounds of different length via subtle changes in the way the substrates are bound. For compounds of four GlcNAc units or larger, the central binding sites (2-5) are likely to be always occupied, in a manner as observed in our structures. However, since X-ray crystallography is an averaging technique it is possible that the binding register may vary, i.e., small populations of, e.g., chitohexaose might be bound with GlcNAc-1 at position 2 (with GlcNAc-6 being disordered). As alluded to above, it is also possible that mixed populations are present with regards to the orientation of the substrate, i.e., the reducing end might be extracellular in part of the population. Future MD simulations could shed light on these details.

Ejection of the pore plug in electrophysiology. To probe the role of the $\mathrm{N}$-terminus in substrate binding and transport in more detail we carried out single-channel electrophysiological experiments for in vitro-folded and OM-expressed VhChiP. Based on the crystal structure we also generated a VhChiP variant with the first $19 \mathrm{~N}$-terminal residues removed, denoted truncated $V h$ ChiP. At standard electrophysiological conditions with applied potentials of -100 and $+100 \mathrm{mV}$, the single-channel conductance is essentially the same $(\sim 2.0 \mathrm{nS})$ for all three $V h$ ChiP proteins (Fig. $7 \mathrm{a}-\mathrm{c}$ ), and are fully consistent with data for the native protein obtained in previous studies ${ }^{10,11}$. Surprisingly, there is no clear evidence of a closed channel for any of the three VhChiP proteins. However, the OM-expressed protein and (to a lesser extent) in vitro-folded ChiP show short-lived channel closures that are absent in the N-terminally truncated channel (Fig. 7c), suggesting infrequent insertions of the plug inside the pore in the presence of a high and non-physiological membrane potential. To probe this further, we first induced closing of the OM-expressed VhChiP channels by applying a high voltage (+199 V; Fig. $7 d)$. We then decreased the voltage to obtain very low membrane potentials $( \pm 2.5 \mathrm{mV}$; Fig. $7 \mathrm{e})$. Under these conditions we observe long-lived channel closures that are fully consistent with the pluginserted state observed in the crystal structure. Interestingly, three subconductance levels are observed corresponding to trimers with one, two or three open pores, indicating that the pore plugs move in and out of the channels independently. The channels open permanently when the voltage is increased to $\pm 10 \mathrm{mV}$ (Fig. 7f). The low voltages required for channel opening are somewhat surprising given the large free energy of plug binding derived from MD simulations (Fig. 3).

A plug peptide blocks the pore of plug-less $V h$ ChiP. To probe the role of the pore plug further, we added a peptide corresponding to the first nine residues of $V h C h i P$ (DGANSDAAK) to the $\mathrm{N}$-terminally truncated protein. At $100 \mu \mathrm{M}$ peptide, frequent current blockages corresponding to the complete closure of a single channel are observed (Fig. 8). No blockages are observed for the control OmpF in the presence of $200 \mu \mathrm{M}$ peptide. Interestingly, blockage of $V h \mathrm{ChiP}$ is asymmetric, i.e., it occurs only upon peptide addition from one side (trans), regardless of the sign of the voltage (Supplementary Fig. 3). Given that proteins are added cis and preferentially insert into the lipid bilayer without translocating the long extracellular loops (i.e., these remain on the cis side), trans most likely corresponds to the periplasmic side of 

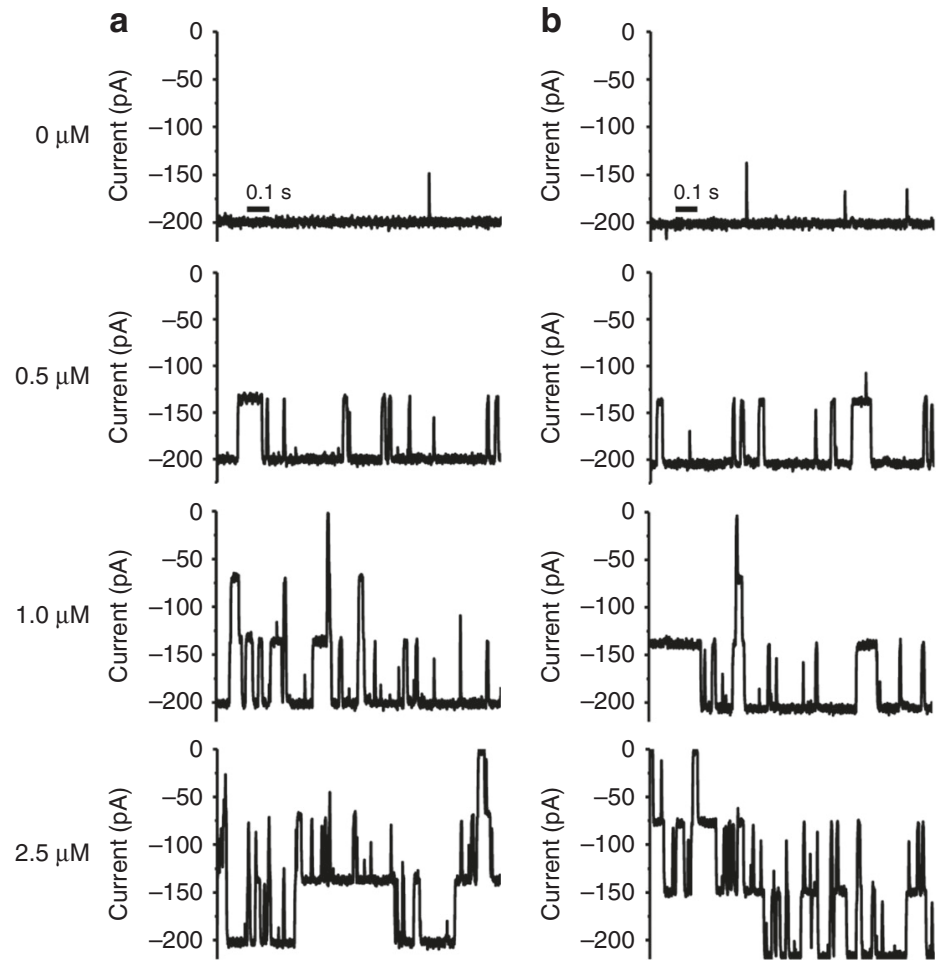
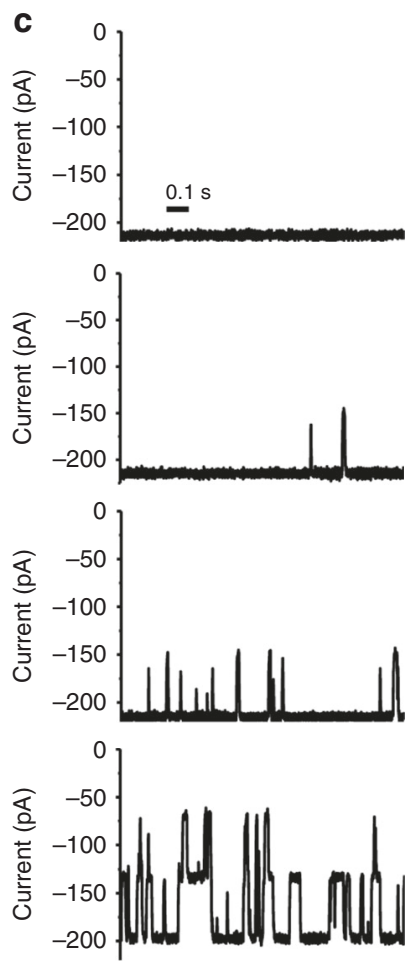

Fig. 9 Chitoxexaose binds to VhChiP with high affinity. Single-channel electrophysiology experiments for natively expressed VhChiP (a), refolded VhChiP (b), and N-terminally truncated VhChiP (c). The fully open VhChiP trimeric channels were exposed to different concentrations of chitohexaose. lon current traces were acquired at $-100 \mathrm{mV}$ with sugar added on the cis side. For full trace recordings of 2 min duration and analyses see Supplementary Figs. 3-6

the channel. This experiment therefore recapitulates the physiological insertion of the pore plug. To further test specificity we also tested two unrelated peptides of similar length for VhChiP pore blockage. Neither of the control peptides affect the VhChiPmediated currents (Supplementary Fig. 4), indicating that pore blockage is specific.

Effect of the N-terminus on substrate binding and transport. Next, we tested the three proteins in the presence of increasing concentrations of chitohexaose (Fig. 9 and Supplementary Figs. 5-8). Under the conditions employed (-100 mV voltage) all three channels are permanently open (previous section). The results show that one subunit of the full-length channels was frequently blocked even at very low concentrations of chitohexaose $(0.5 \mu \mathrm{M})$, while under the same conditions blocking events were rarely seen with the truncated channel. At $2.5 \mu \mathrm{M}$, occlusion of all three subunits was observed for the full-length channels, but not for truncated VhChiP. The data therefore suggest that the truncated channel interacts weaker with the sugar compared to the full-length channels. When the equilibrium binding constant $K$ is evaluated, the full-length channels show relatively similar binding constants of $\sim 2-5 \times 10^{5} \mathrm{M}^{-1}$ in agreement with previous results (Supplementary Table 2$)^{11,28}$. The binding constants vary somewhat with different membrane potentials and are generally two- to threefold higher for the in vitro-folded protein for reasons that are not clear. However, removal of the first nine residues affects substrate binding significantly, with binding constants 10-15-fold lower for truncated VhChiP compared to OMexpressed, full-length protein (Supplementary Table 2).

For most systems, a limitation of single-channel electrophysiology is the inability to distinguish substrate binding from substrate translocation through the channel, i.e., a compound can be released on the same side as where it binds. We therefore also assayed in vitro transport of various sugars via proteoliposome swelling assays. Various monomeric sugars translocate efficiently through VhChiP in the presence and absence of the $\mathrm{N}$-terminus (Fig. 10). The truncated variant exhibited a more non-specific channel behavior and was approximately twofold more efficient in transport of various types of small monosaccharides compared to the full-length channels. By contrast, translocation of oligomeric sugars is exquisitely specific in accordance with previous results ${ }^{10,11}$, i.e., only chito-oligosaccharide substrates are translocated efficiently. Interestingly, for the oligosaccharides the absence of the $\mathrm{N}$-terminus leads to a substantial decrease in transport rates, which is in qualitative agreement with the decreased binding constants observed in electrophysiology. Collectively the results suggest that the $\mathrm{N}$-terminal segment plays an important role in chito-oligosaccharide substrate translocation through the VhChiP channel (Fig. 10).

\section{Discussion}

The X-ray crystal structure of trimeric OM-purified VhChiP shows an unprecedented closing of the transport pore by the $\mathrm{N}$ terminus of a neighboring monomer. Potential of mean force calculations suggest that this structural feature is highly stable in the absence of substrate. However, the applied-field MD simulations do not explain why the N-terminal plug is ejected at very low voltages in the experiments. These ejections might be rather slow processes involving details of the ion distributions and electro-osmotic forces on the plug that are currently out of scope for the present MD simulations due to the limited simulation time scales. In addition, the apparent discrepancy between the high computed binding free-energy of the plug and the measured low voltage of plug ejection might be due to differences in salt conditions, constant protonation states in the simulations and force-field approximations.

Interestingly, the single-channel experiments on truncated VhChiP in the presence of chitohexaose suggest that the absence 

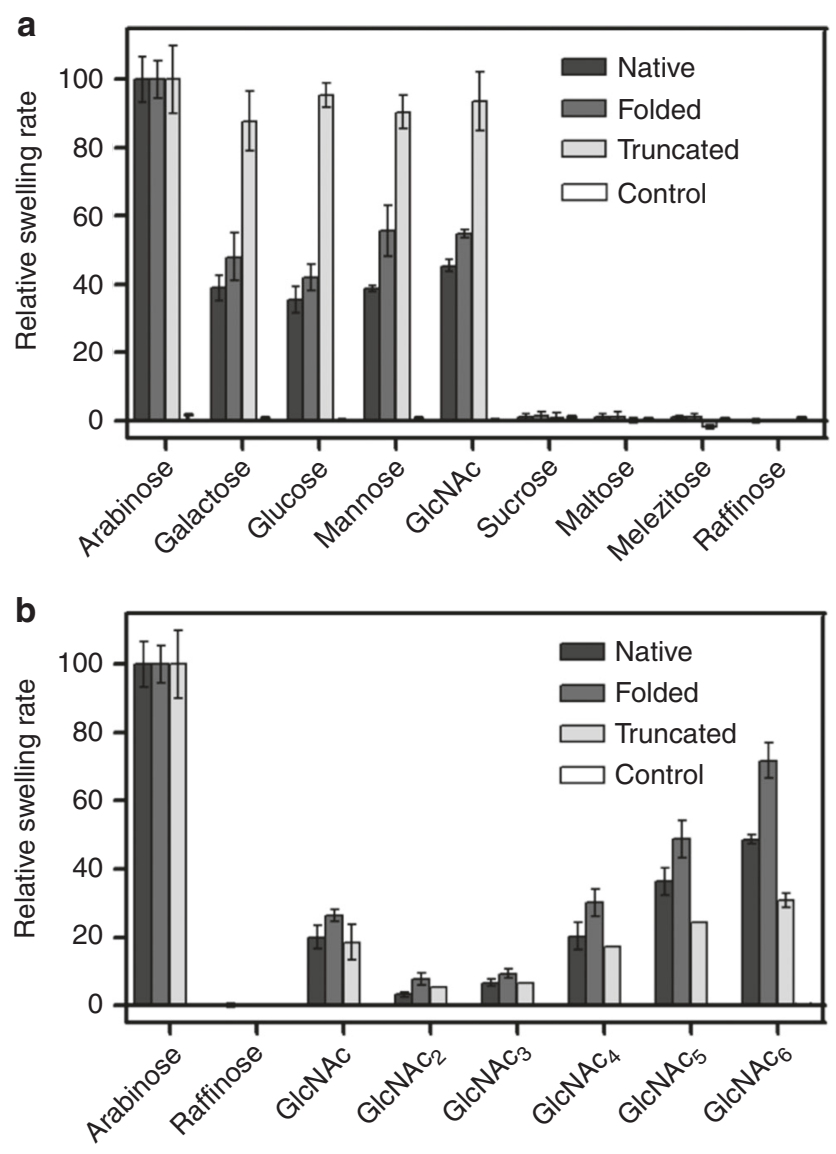

Fig. 10 The $\mathrm{N}$-terminus is important for chito-oligosaccharide substrate transport. Proteoliposome swelling assays in the presence of various monoand oligosaccharides (a) as well as chito-oligosacharides of different lengths (b). No permeation of any sugars was observed when tested with control liposomes without VhChiP. "Folded" denotes in vitro-folded VhChiP. Data represent mean \pm s.d., $n=3$. For details see Methods

of the N-terminus results in a weaker binding affinity of the substrate for the channel. Likewise, the proteoliposome swelling experiments show lower rates of chitooligosaccharide transport in the VhChiP variant lacking the $\mathrm{N}$-terminus. These results have several implications. First, they would be difficult to reconcile with a scenario where the $\mathrm{N}$-terminus would always be disordered and located outside the barrel, as observed in the crystal structure obtained from in vitro-folded VhChiP. Thus, the results suggest that N-terminal gating also occurs in the lipid bilayer, which is confirmed by single-channel experiments at very low membrane potential, approaching physiological conditions where the Donnan potential across the OM will be close to zero ${ }^{36}$. Second, the $\mathrm{N}$-terminal gate is most likely not a passive structural feature but instead promotes substrate translocation. How this occurs is still unclear, but one possibility is that the $\mathrm{N}$-terminal plug generates the optimal configuration of the substrate binding site. This hypothesis is given some support by the MD simulations, which show that the presence of the $\mathrm{N}$-terminus results in increased dynamics of a region in loop L3 close to several substrateinteracting residues (Fig. 3a). This could be beneficial to substrate binding/orientation and consequently would make transport more efficient, providing support for the electrophysiology and in vitro transport data.

Is the putative gating mechanism conserved and why would there be a need for gating? Inspection of the sequence alignment (Fig. 5) shows that the N-terminal extension forming the pore plug is present only in a subset of VhChiP orthologs from Vibrio spp., indicating that other VhChiP orthologs may not be gated, or perhaps are gated in a different way. Regarding the need for a gating mechanism, in other enteric bacteria such as $E$. coli it is well established that the general porins $\mathrm{OmpF}$ and $\mathrm{OmpC}$ are not gated, but differently expressed depending on the osmolarity of the medium. At high osmolarity, expression of the smallerdiameter OmpC is upregulated relative to that of OmpF, presumably restricting the salt concentrations in the periplasmic space. Vibrio spp. also have orthologs of the general porins (named OmpU and OmpT in $V$. cholerae). While the structures of OmpU and OmpT have not yet been solved it is known that their expression is also regulated by osmolarity, with the largerpore OmpU protein downregulated under conditions of high osmolarity $^{37}$. Thus, a general picture emerges where under conditions of high osmolarity the entry of excess ions is restricted via upregulation of smaller-diameter channels and/or downregulation of larger ones. VhChiP is highly upregulated in marine environments in the presence of chitin ${ }^{21}$; for such a highly expressed channel, gating would be an effective means to restrict the entry of ions in a high-osmolarity environment. As mentioned above, how VhChiP orthologs with a shorter N-terminus (Fig. 5) would restrict ion access to the periplasmic space is not yet clear.

In conclusion, our studies on an important OM channel from marine Vibrio spp. highlight an emerging theme that substrate transport by OM channels is often more complicated than simple diffusion through a large, static pore. Ligand gating has been observed before in monomeric 14-stranded OM proteins ${ }^{33,38}$, but gating by a neighbor within an oligomeric assembly has, to our knowledge, not been reported in any membrane protein. Moreover, our results demonstrate that gating, rather than being a passive switch between open and closed states, might play a more active role in substrate transport. Future studies will be required to illuminate how gating improves substrate translocation and how ligands cause the necessary conformational changes that open the gate.

\section{Methods}

Cloning and expression of OM-expressed VhChiP. A BlastP search with chitoporin from $V$. furnissii as input (UniProtKB/TrEMBL entry: Q9KK91) identified VIBHAR_01269 as a putative chitoporin ortholog (GenBANK accession number YP_001444474) from the $V$. harveyi type strain ATCC BAA-1116 (also named $V$. campbellii ATCC BAA-1116). Therefore, oligonucleotides were designed for the gene from the BAA-1116 BB120 strain to clone the chitoporin gene from our laboratory strain ( $V$. harveyi type strain 650$)$. Genomic DNA was prepared using the PureLink Genomic DNA Kit (Invitrogen, Gibthai Company Ltd., Bangkok, Thailand) and used as the template for PCR. The oligonucleotides used for PCR are listed in Supplementary Table 3. The PCR product was of the expected size (1.1 $\mathrm{kbp})$ and was cloned into pET23d(+) using Nco I and Xho I restriction sites, following the protocol of the manufacturer. Nucleotide sequences of both strands of the PCR product were determined by automated sequencing (First BASE Laboratories Sdn Bhd, Selangor Darul Ehsan, Malaysia). The recombinant plasmid harboring the chiP gene (pET23d(+)/chiP) was transformed into E. coli BL21(DE3) omp8 rosetta $\left(\Delta l a m B\right.$ ompF::Tn5 5 ompA $\Delta o m p C^{39}$ ) (a gift from Prof. Roland Benz, Jacob University Bremen, Germany). The transformed cells were grown at $37^{\circ} \mathrm{C}$ in Luria-Bertani (LB) liquid medium containing $100 \mu \mathrm{g} \mathrm{mL}^{-1}$ ampicillin, $25 \mu \mathrm{g} \mathrm{mL}^{-1}$ kanamycin and $1 \%(\mathrm{w} / \mathrm{v})$ glucose. The expression was induced by adding $0.4 \mathrm{mM}$ IPTG at an $\mathrm{OD}_{600} \sim 0.6$ and the cells were incubated for $6 \mathrm{~h}$ at $37^{\circ} \mathrm{C}$. After cell disruption, the membranes were spun down and incubated with $2 \%(\mathrm{w} / \mathrm{v})$ SDS for $1 \mathrm{~h}$ at $50{ }^{\circ} \mathrm{C}$. Afterwards the membranes were washed with $0.125 \%(\mathrm{v} / \mathrm{v})$ octyl-POE and finally extracted with $3 \%(\mathrm{v} / \mathrm{v})$ octyl-POE. Insoluble particles were centrifuged for $30 \mathrm{~min}$ at $235,000 \times g$ and the supernatant was loaded onto a HiPrep Mono Q anion exchange column. The column was washed with 10 column volumes (CV) $20 \mathrm{mM}$ (Phosphate buffer) sodium phosphate and $0.2 \%$ (v/v) LDAO at pH 7.4. The protein was eluted with a linear gradient of $0-1 \mathrm{M} \mathrm{KCl}$. Fractions containing VhChiP were pooled and subjected to size exclusion chromatography using a HiLoad 16/600 superdex 200 (GE Healthcare) using $10 \mathrm{mM}$ HEPES, $100 \mathrm{mM} \mathrm{LiCl}$ $0.4 \%(\mathrm{v} / \mathrm{v}) \mathrm{C}_{8} \mathrm{E}_{4}, \mathrm{pH} 7.5$. The purified protein was concentrated to $10-12 \mathrm{mg} \mathrm{mL}^{-1}$ and directly flash-frozen into liquid nitrogen prior to setting up crystallization trials.

The gene encoding for truncated $V h C h i P$, lacking the first nineteen residues of the mature sequence (EVYGII--), was synthesized by GenScript and cloned into 
pET23a (Novagen). Subsequent protein expression and purification was carried out as described above for the full-length protein.

Inclusion body expression of SeMet-labeled VhChiP. For expression of VhChiP into IBs, the gene without signal sequence and His-tag was cloned into pET28a (Novagen) via $\mathrm{NcoI}$ and $\mathrm{XhoI}$ (Supplementary Table 3). This procedure added the sequence "MG" to the $\mathrm{N}$-terminus of the mature protein (MGDGANS--). The plasmid was transformed into BL21(DE3) cells (New England Biolabs). The cells were grown in minimal media (LeMasters-Richards) to $\mathrm{OD}_{600} \sim 0.6$ at $37^{\circ} \mathrm{C}$ before SeMet in combination with lysine, phenylalanine, threonine, leucine, isoleucine, and valine were added. Half an hour later the cells were induced with $1 \mathrm{mM} \mathrm{IPTG}$ at $37^{\circ} \mathrm{C}$ for $3 \mathrm{~h}$. Cells were harvested by centrifugation at $4500 \times g$ for $30 \mathrm{~min}$ (Beckman Coulter). After cell disruption, IBs were harvested by centrifugation at $12,000 \times g$ for $20 \mathrm{~min}$ and washed once in TBS with $1 \%$ Triton X-100 for $30 \mathrm{~min}$ at room temperature. This was followed by two washes without Triton X-100, followed by centrifugation for $20 \mathrm{~min}$ at $12,000 \times g$ after each step. IBs were then solubilized at room temperature by stirring in $\sim 25 \mathrm{~mL}$ TBS with $8 \mathrm{M}$ Urea for $2 \mathrm{~h}$. Non-solubilized pellet was removed by centrifugation at $200,000 \times g$ for $30 \mathrm{~min}$. For in vitro folding, the supernatant $(\sim 20 \mathrm{~mL})$ was added dropwise to $200 \mathrm{~mL}$ TBS including 3\% Elugent (Calbiochem) and the folding reaction was allowed to proceed at room temperature overnight with slow stirring. Subsequently, the folded protein was applied to a $10 \mathrm{~mL}$ anion exchange column and purified as described above for the OM-expressed protein.

\section{Crystallization and structure solution. Initial crystallization trials for OM-} expressed $V h$ ChiP $\left(10 \mathrm{mg} \mathrm{mL}^{-1}\right)$ were performed at $295 \mathrm{~K}$ by sitting-drop vapor diffusion using MemGold1- and MemGold2-Screen from Molecular Dimensions with a mosquito robot (TTP Labtech). The initial hits were optimized by finescreening with larger drops by hanging drop vapor diffusion. Crystals in space group C2 were grown in 28\% (w/v) PEG 400, $0.2 \mathrm{M}$ sodium acetate, $0.1 \mathrm{M} \mathrm{MES} \mathrm{pH}$ 6.5. Crystals were directly flash-frozen in liquid nitrogen. A data set was collected at IO2 at the Diamond Light Source (DLS), UK. Se-Met crystals, derived from in vitro-folded $V h$ ChiP $\left(\sim 12 \mathrm{mg} \mathrm{mL}^{-1}\right)$, were obtained in two different crystal forms. Crystal form I in space group P2 ${ }_{1}$ was crystallized in $30 \%$ (w/v) PEG $400,0.05 \mathrm{M}$ $\mathrm{NaCl}, 0.1 \mathrm{M}$ sodium citrate $\mathrm{pH}$ 5.5. Crystals in crystal form II were grown in $28 \%$ (w/v) PEG 400, 0.5 M potassium iodide, 0.1 M Tris $\mathrm{pH}$ 8.5. A SAD data set for crystal form I was collected to $1.95 \AA$ at beamline IO2 at the Diamond Light Source (DLS), UK. Data were integrated and scaled with $\mathrm{XDS}^{40}$. Initial phasing and modeling was done using AUTOSOL within PHENIX ${ }^{41}$. Further model building was performed using the program $\mathrm{COOT}^{42}$. The protein model was refined with REFMAC $^{43}$. Phases for OM-expressed VhChiP and in vitro-folded VhChiP in crystal form II were obtained by MR using MOLREP ${ }^{44}$ with the refined structure of SeMet-VhChiP as a search model. Model building was performed using $\mathrm{COOT}^{42}$ and the structure was refined with REFMAC ${ }^{43}$. The program MolProbity ${ }^{45}$ was used to evaluate the final model and $\mathrm{PyMOL}^{46}$ (Schrödinger, LLC) for the visualization of the protein structures.

For VhChiP complex structures, in vitro-folded protein $\left(12 \mathrm{mg} \mathrm{mL}^{-1}\right)$ was cocrystallized with $2.5 \mathrm{mM} \mathrm{GlcNAc}_{6}$ in $34 \%$ (w/v) PEG $400,0.05 \mathrm{M} \mathrm{NaCl}, 0.1 \mathrm{M}$ sodium citrate $\mathrm{pH}$ 5.5. Accordingly, OM-expressed protein $\left(10 \mathrm{mg} \mathrm{mL}^{-1}\right)$ was cocrystallized with $10 \mathrm{mM} \mathrm{GlcNAc}$ in $28 \%$ (w/v) PEG 400, $0.2 \mathrm{M}$ sodium acetate, $0.1 \mathrm{M}$ MES $\mathrm{pH}$ 6.5. The structures were solved via MR as described above for the apo proteins.

MD simulations. The starting structures of the MD simulations were prepared as follows. The crystal structures of the VhChiP trimer in closed and open conformations were embedded in a 1-Palmitoyl-2-oleoyl-sn-glycero-3-phosphoethanolamine (POPE) bilayer together with TIP3P water and $1 \mathrm{M} \mathrm{KCl}$ salt.

Subsequently, an energy minimization was performed using the steepest descent algorithm for 5000 steps followed by a 10 ns equilibration in the NVT and NPT ensembles with positional restraints on the heavy atoms of the proteins and lipids. The final unbiased simulations of VhChiP systems in open and closed conformations were performed for $500 \mathrm{~ns}$. Furthermore, we carried out applied-field simulations for the in vitro-folded channel $(200 \mathrm{mV}: 3 \times 250 \mathrm{~ns}$ each) and OMexpressed channel $(200 \mathrm{mV}: 3 \times 250 \mathrm{~ns}$ each). For the free-energy calculations, we built an in silico VhChiP trimer variant with one mutation per monomer (D1A, D6A, and K9A) and a wild type system, both in the plug-inserted conformation, and including the necessary number of potassium ions to neutralize the system.

All MD simulations were performed with the GROMACS 4.6.5 program $^{47}$ using the CHARMM36 all-atom force field ${ }^{48,49}$. The particle-mesh Ewald approach was used to calculate the long-range electrostatic interactions with a cutoff of $12 \AA$. Short-range Coulomb and Lennard Jones interactions were explicitly calculated up to the cutoff distance. The LINCS algorithm was applied to constraint the lengths of all bonds containing hydrogen atoms ${ }^{50}$. Moreover, the unbiased simulations discussed in this study were carried out in the NPT ensemble achieved by a semiisotropic Parrinello-Rahman barostat ${ }^{51}$ at 1 bar with a coupling constant of $5 \mathrm{ps}$ and the Nosé-Hoover thermostat ${ }^{52,53}$ with a coupling constant of 1 ps. The applied-field simulations were performed in a NVT ensemble with an electric field corresponding to transmembrane potential of $200 \mathrm{mV}$ towards the periplasmic side of the channel. The field strength is proportional to the voltage $V$, i.e., $E=V / L_{z}$, where $L_{z}$ denotes the system length in $z$ direction normal to the membrane. During the MD simulations snapshots were collected at every $10 \mathrm{ps}$ and the MD results were analyzed using the tools available in GROMACS 4.6.5 package.

To estimate the energetics for unbinding of the $\mathrm{N}$-terminus, two umbrella sampling calculations were performed for the wild type and the above-described trimer variant system. To save computational resources, we took advantage of the trimeric organization of the channel and simultaneously simulated the free energy profiles of the three mutant plugs (D1A, D6A, and K9A) along a single reaction coordinate. The one-dimensional reaction coordinate corresponded to the $z$ coordinate distance between the centers of mass (COM) of the N-terminal plug (C $\alpha$ atoms of residues 1-10) and the corresponding barrel ( $\mathrm{C} \alpha$ atoms of residues $25-350)$. The reaction coordinate was divided into 28 windows and we simulated each window for $90 \mathrm{~ns}$ with a harmonic potential of $1000 \mathrm{~kJ} \mathrm{~mol}^{-1} \mathrm{~nm}^{-2}$ on the reaction coordinate. The unbiased free energy profiles were calculated from the last $80 \mathrm{~ns}$ of each window trajectory using the g_wham ${ }^{54}$ implementation of the weighted histogram analysis method ${ }^{55}$.

Single-channel electrophysiology. Planar lipid bilayer reconstitution was carried out in electrolyte containing $1 \mathrm{M} \mathrm{KCl}$ and $20 \mathrm{mM}$ HEPES pH 7.5 , at $25^{\circ} \mathrm{C}^{28,56}$. Montal-Mueller type solvent-free bilayer formation was carried out using 1,2diphytanoyl-sn-glycero-3-phosphatidylcholine (DPhPC; Avanti Polar Lipids, Alabaster, AL). First, the aperture was pre-painted with a few microliters of $1 \%(\mathrm{v} / \mathrm{v})$ hexadecane in hexane, after which a planar bilayer was formed across the aperture by lowering and raising the liquid level. Ion currents were detected using $\mathrm{Ag} / \mathrm{AgCl}$ electrodes with a patch-clamp amplifier connected to a two-electrode bilayer headstage (PC-ONE plus PC-ONE-50; Dagan Corp., Minneapolis, MN, USA). The bilayer setup was operated within a Faraday cage on a vibration-dampened table, with an A/D converter (LIH 1600, HEKA Elektronik, Lambrecht, Germany) and was operated using the PULSE software program (HEKA Elektronik, Lambrecht, Germany). One of the electrodes, immersed in $1 \mathrm{M} \mathrm{KCl} \mathrm{electrolyte} \mathrm{on} \mathrm{the} \mathrm{cis} \mathrm{side} \mathrm{of}$ the cuvette, was connected to ground, while the electrode on the trans side was connected to the amplifier head-stage. VhChiP was always added to the cis side of the cuvette. Conductance values were determined from the $I-V$ curves obtained from single-channel insertions at different voltages.

To investigate sugar translocation, single channels of the $V h C h i P$ variants were reconstituted in the artificial lipid membrane. To prevent multiple insertions during data acquisition, the protein solution in the chamber was gently diluted after the first insertion by sequential additions of working electrolyte. Subsequently the fully open channel was titrated with chitohexaose with concentrations ranging from 0.1 to $10 \mu \mathrm{M}$. Sugars were added to the cis side of the chamber. Ion flow was usually recorded for $2 \mathrm{~min}$ at different transmembrane potentials. The equilibrium binding constant $K\left(\mathrm{M}^{-1}\right)$ was derived from the decrease in the ion conductance resulting from increasing concentrations of sugar using the following Equation (1) (ref. 57):

$$
G_{\max }-G_{c} / G_{\max }=I_{\max }-I_{c} / I_{\max }=K[c] /(K[c]+1),
$$

$G_{\max }$ is the average conductance of the fully open $V h$ ChiP channel and $G_{c}$ is the average conductance at a given concentration $[c]$ of a chitooligosaccharide. $I_{\max }$ is the initial current through the fully open channel without sugar and $I_{\mathrm{c}}$ is the current at a particular sugar concentration.

Liposome swelling experiments. VhChiP-reconstituted proteoliposomes were prepared using soybean $\mathrm{L}-\alpha$-phosphatidylchloline $\left(20 \mathrm{mg} \mathrm{mL}^{-1}\right.$, freshly prepared in chloroform) (Sigma-Aldrich) to form multi-lamellar liposomes ${ }^{10}$. Following this, $200 \mathrm{ng}$ of $\mathrm{VhChiP}$ was reconstituted into $200 \mu \mathrm{L}$ of liposome suspension by sonication, and $17 \%(\mathrm{w} / \mathrm{v})$ dextran $(40 \mathrm{kDa})$ was entrapped in the proteoliposomes by drying under vacuum and resuspending in $10 \mathrm{mM}$ phosphate buffer $\mathrm{pH}$ 7.5. For determination of the isotonic solute concentration, D-Raffinose solutions were prepared in phosphate buffer with concentrations of $40,50,60$, and $70 \mathrm{mM}$. The obtained isotonic concentration was then used for the adjustment of the isotonic concentration for other solutes. All small sugars (Fig. 10a) were prepared at $60 \mathrm{mM}$ concentration. For chitooligosaccharides (Fig. 10b) we prepared the solutions differently, and used $1 \mathrm{mM}$ of each chitosugar in $59 \mathrm{mM}$ raffinose. The osmolarity of each sugar solution was checked to be at the isotonic concentration using a Genotec Osmometer 300. The reasons for using low concentrations of the chitosugars are twofold: (i) $\mathrm{GlcNAc}_{4-6}$ are not soluble at $60 \mathrm{mM}$ and (ii) $\mathrm{GlcNAc}_{4-6}$ interact with $V h C h i P$ at low $\mu \mathrm{M}$ concentration (not $\mathrm{mM}$ ). The liposome-swelling assays were carried out by adding $25 \mu \mathrm{L}$ of proteoliposome suspension to $600 \mu \mathrm{L}$ of sugar solution and the changes in absorbance at $500 \mathrm{~nm}$ were monitored immediately. The absorbance change over the first $60 \mathrm{~s}$ was used to estimate the swelling rate $\left(\mathrm{s}^{-1}\right)$ according to: $\varphi=\left(1 / A_{i}\right) \mathrm{d} A / \mathrm{d} t$, in which $\varphi$ is the swelling rate, $A_{i}$ the initial absorbance, and $\mathrm{d} A / \mathrm{d} t$ the rate of absorbance change during the first $60 \mathrm{~s}$. The swelling rate for each sugar was normalized by setting the rate of $\mathrm{L}$-arabinose $(150$ $\mathrm{Da}$ ), the smallest sugar, to $100 \%$. The values presented are averages from three to five independent experiments. The sugars tested were D-glucose $(180 \mathrm{Da}), \mathrm{D}-$ mannose (180 Da), D-galactose (180 Da), $N$-acetylglucosamine (GlcNAc) $(221 \mathrm{Da})$, D-sucrose (342 Da), D-melezitose (522 Da), GlcNAc 2 (424 Da), GlcNAc 3 (628 Da), $\mathrm{GlcNAc}_{4}(830 \mathrm{Da}), \mathrm{GlcNAc}_{5}(1034 \mathrm{Da}), \mathrm{GlcNAc}_{6}(1237 \mathrm{Da})$, and maltodextrins. 
Protein-free liposomes and proteoliposomes without sugars were used as negative controls.

Data availability. The atomic coordinates and the associated structure factors have been deposited in the Protein Data Bank (http://www.pdbe.org) with accession codes $5 \mathrm{MDO}$ (In vitro-folded VhChiP, crystal form 1), 5MDP (In vitro-folded VhChiP, crystal form 2), 5MDQ (OM-expressed VhChiP), 5MDR (In vitro-folded $V h C h i P$ with chito-hexaose), and 5MDS (OM-expressed VhChiP with chito-tetraose). Other data supporting the findings of this study are available from the corresponding authors upon reasonable request.

Received: 8 May 2017 Accepted: 7 December 2017 Published online: 15 January 2018

\section{References}

1. Klemm, D. et al. Cellulose: fascinating biopolymer and sustainable raw material. Angew. Chem. Int. Ed. 44, 3358-3393 (2005).

2. Muzzarelli, R. A. A. Chitin (Pergamon Press, UK, 1977).

3. Gooday, G. W. The ecology of chitin degradation. Adv. Microb. Ecol. 11, $387-430$ (1990).

4. Tracey, M. A. Chitin. Rev. Pure Appl. Chem. 7, 1-13 (1957).

5. Hirono, I., Yamashita, M. \& Aoki, T. Note: molecular cloning of chitinasegenes from Vibrio anguillarum and V. parahaemolyticus. J. Appl. Microbiol. 84, 1175-1178 (1998).

6. Zobell, C. E. \& Rittenberg, S. C. The occurrence and characteristics of chitinoclastic bacteria in the sea. J. Bacteriol. 35, 275-287 (1938).

7. Hunt, D. E. et al. Conservation of the chitin utilization pathway in the Vibrionaceae. Appl. Environ. Microbiol. 74, 44-51 (2008).

8. Jung, B. O., Roseman, S. \& Park, J. K. The central concept for chitin catabolic cascade in marine bacterium, Vibrios. Macromol. Res. 16, 1-15 (2008).

9. Keyhani, N. O., Li, X. B. \& Roseman, S. Chitin catabolism in the marine bacterium Vibrio furnissii. Identification and molecular cloning of a chitoporin. J. Biol. Chem. 275, 33068-33076 (2000).

10. Suginta, W. et al. Molecular uptake of chitooligosaccharides through chitoporin from the marine bacterium Vibrio harveyi. PLoS ONE 8, e55126 (2013).

11. Suginta, W. et al. Chitoporin from Vibrio harveyi, a channel with exceptional sugar specificity. J. Biol. Chem. 288, 11038-11046 (2013).

12. Keyhani, N. O. \& Roseman, S. The chitin catabolic cascade in the marine bacterium Vibrio furnissii. Molecular cloning, isolation, and characterization of a periplasmic chitodextrinase. J. Biol. Chem. 271, 33414-33424 (1996).

13. Suginta, W. et al. Novel $\beta$-N-acetylglucosaminidases from Vibrio harveyi 650: cloning, expression, enzymatic properties, and subsite identification. $B M C$ Biochem. 11, 40 (2010).

14. Keyhani, N. O. \& Roseman, S. The chitin catabolic cascade in the marine bacterium Vibrio furnissii. Molecular cloning, isolation, and characterization of a periplasmic beta-N-acetylglucosaminidase. J. Biol. Chem. 271, 33425-33432 (1996).

15. Bassler, B. L. et al. Chitin utilization by marine bacteria. Chemotaxis to chitin oligosaccharides by Vibrio furnissii. J. Biol. Chem. 266, 24268-24275 (1991).

16. Keyhani, N. O. et al. The chitin catabolic cascade in the marine bacterium Vibrio furnissii. Characterization of an $\mathrm{N}, \mathrm{N}^{\prime}$-diacetyl-chitobiose transport system. J. Biol. Chem. 271, 33409-33413 (1996).

17. Bouma, C. L. \& Roseman, S. Sugar transport by the marine chitinolytic bacterium Vibrio furnissii. Molecular cloning and analysis of the glucose and Nacetylglucosamine permeases. J. Biol. Chem. 271, 33457-33467 (1996).

18. Park, J. K., Keyhani, N. O. \& Roseman, S. Chitin catabolism in the marine bacterium Vibrio furnissii. Identification, molecular cloning, and characterization of A N,N'-diacetylchitobiose phosphorylase. J. Biol. Chem. 275, 33077-33083 (2000)

19. Li, X. \& Roseman, S. The chitinolytic cascade in Vibrios is regulated by chitin oligosaccharides and a two-component chitin catabolic sensor/kinase. Proc. Natl Acad. Sci. USA 101, 627-631 (2004).

20. Suginta, W. et al. An endochitinase A from Vibrio carchariae: cloning, expression, mass and sequence analyses, and chitin hydrolysis. Arch. Biochem. Biophys. 424, 171-180 (2004).

21. Meibom, K. L. et al. The Vibrio cholerae chitin utilization program. Proc. Natl Acad. Sci. USA 101, 2524-2529 (2004).

22. Schmitt, E. K., Vrouenraets, M. \& Steinem, C. Channel activity of OmpF monitored in nano-BLMs. Biophys. J. 91, 2163-2171 (2006).

23. Benz, R. et al. Pore formation by LamB of Escherichia coli in lipid bilayer membranes. J. Bacteriol. 165, 978-986 (1986).

24. Schülein, K. \& Benz, R. LamB (maltoporin) of Salmonella typhimurium: isolation, purification and comparison of sugar binding with LamB of Escherichia coli. Mol. Microbiol. 4, 625-632 (1990).
25. Suginta, W. \& Smith, M. F. Single-molecule trapping dynamics of sugar-uptake channels in marine bacteria. Phys. Rev. Lett. 110, 238102 (2013).

26. Suginta, W., Winterhalter, M. \& Smith, M. F. Correlated trapping of sugar molecules by the trimeric protein channel chitoporin. Biochim. Biophys. ActaBiomembr. 1858, 3032-3040 (2016).

27. Zachariae, U. et al. High resolution crystal structures and molecular dynamics studies reveal substrate binding in the porin Omp32. J. Biol. Chem. 281, 7413-7420 (2006).

28. Chumjan, W. et al. Chitoporin from the marine bacterium Vibrio harveyi: probing the essential roles of Trp136 at the surface of the constriction zone. $J$ Biol. Chem. 290, 19184-19196 (2015).

29. Moraes, T. F. et al. An arginine ladder in OprP mediates phosphate-specific transfer across the outer membrane. Nat. Struct. Mol. Biol. 14, 85-87 (2007).

30. Hayat, S. et al. Inclusion of dyad-repeat pattern improves topology prediction of transmembrane $\beta$-barrel proteins. Bioinformatics 32, 1571-1573 (2016).

31. Tsirigos, K. D., Elofsson, A. \& Bagos, P. G. PRED-TMBB2: improved topology prediction and detection of beta-barrel outer membrane proteins. Bioinformatics 32, i665-i671 (2016).

32. Holm, L. \& Rosenstrom, P. Dali server: conservation mapping in 3D. Nucleic Acids Res. 38, W545-W549 (2010)

33. van den Berg, B. et al. Outer-membrane translocation of bulky small molecules by passive diffusion. Proc. Natl Acad. Sci. USA 112, E2991-E2999 (2015).

34. Dutzler, R., Wang, Y. F., Rizkallah, P., Rosenbusch, J. P. \& Schirmer, T. Crystal structures of various maltooligosaccharides bound to maltoporin reveal a specific sugar translocation pathway. Structure 4, 127-134 (1996).

35. van Straaten, K. E. et al. Structure of Escherichia coli lytic transglycosylase MltA with bound chitohexaose: implications for peptidoglycan binding and cleavage. J. Biol. Chem. 282, 21197-21205 (2007).

36. Sen, K., Hellman, J. \& Nikaido, H. Porin channels in intact cells of Escherichia coli are not affected by Donnan potentials across the outer membrane. J. Biol. Chem. 263, 1182-1187 (1988).

37. Chakrabarti, S. R. et al. Porins of Vibrio cholerae: purification and characterization of OmpU. J. Bacteriol. 178, 524-530 (1996).

38. Lepore, B. W. et al. Ligand-gated diffusion across the bacterial outer membrane Proc. Natl Acad. Sci. USA 108, 10121-10126 (2011).

39. Prilipov, A., Phale, P. S., Van Gelder, P., Rosenbusch, J. P. \& Koebnik, R. Coupling site-directed mutagenesis with high-level expression: large scale production of mutant porins from E. coli. FEMS Microbiol. Lett. 163, 65-72 (1998).

40. Kabsch, W. Integration, scaling, space-group assignment and post-refinement. Acta Crystallogr. D Biol. Crystallogr. 66, 133-144 (2010).

41. Adams, P. D. et al. PHENIX: a comprehensive Python-based system for macromolecular structure solution. Acta Crystallogr. D Biol. Crystallogr. 66 , 213-221 (2010).

42. Emsley, P. \& Cowtan, K. Coot: model-building tools for molecular graphics. Acta Crystallogr. D Biol. Crystallogr. 60, 2126-2132 (2004).

43. Murshudov, G. N. et al. REFMAC5 for the refinement of macromolecular crystal structures. Acta Crystallogr. D Biol. Crystallogr. 67, 355-367 (2011).

44. Vagin, A. \& Teplyakov, A. MOLREP: an automated program for molecular replacement. J. Appl. Crystallogr. 30, 1022-1025 (1997).

45. Chen, V. B. et al. MolProbity: all-atom structure validation for macromolecular crystallography. Acta Crystallogr. D Biol. Crystallogr. 66, 12-21 (2010).

46. The PyMOL Molecular Graphics System, v.1.8 (Schrödinger, LLC., 2017)

47. Pronk, S. et al. Gromacs 4.5: a high-throughput and highly parallel open source molecular simulation toolkit. Bioinformatics 29, 845-854 (2013).

48. Klauda, J. B. et al. Update of the CHARMM all-atom additive force field for lipids: validation on six lipid types. J. Phys. Chem. B 114, 7830-7843 (2010).

49. Best, R. B. et al. Optimization of the additive CHARMM All-atom protein force field targeting improved sampling of the backbone $\varphi, \psi$ and Side-chain $\chi 1$ and $\chi 2$ dihedral angles. J. Chem. Theory Comput. 8, 3257-3273 (2012).

50. Hess, B., Bekker, H., Berendsen, H. J. C. \& Johannes, G. E. M. F. LINCS: a linear constraint solver for molecular simulations. J. Comput. Chem. 18, 1463-1472 (1997).

51. Parrinello, M. \& Rahman, A. Polymorphic transitions in single crystals: a new molecular dynamics method. J. Appl. Phys. 52, 7182-7190 (1981).

52. Nosé, S. A. Molecular dynamics method for simulations in the canonical ensemble. Mol. Phys. 52, 255-268 (1984).

53. Hoover, W. G. Canonical dynamics: equilibrium phase-space distributions. Phys. Rev. A 31, 1695 (1985).

54. Hub, Jochen S., de Groot, B. L. \& van der Spoel, D. g_whams: a free weighted histogram analysis implementation including robust error and autocorrelation estimates. J. Chem. Theory Comput. 6, 3713-3720 (2010).

55. Kumar, S., Bouzida, D., Swendsen, R. H., Kollman, P. A. \& Rosenberg, J. M. The weighted histogram analysis method for free-energy calculations on biomolecules. I. The method. J. Comput. Chem. 13, 1011-1021 (1992).

56. Schulte, A. et al. The outer membrane protein VhOmp of Vibrio harveyi: poreforming properties in black lipid membranes. J. Membr. Biol. 230, 101-111 (2009). 
57. Benz, R. \& Hancock, R. E. Mechanism of ion transport through the anionselective channel of the Pseudomonas aeruginosa outer membrane. J. Gen. Physiol. 89, 275-295 (1987).

\section{Acknowledgements}

B.v.d.B. would like to acknowledge the Royal Society for salary support. We are also indebted to the staff at beam lines I02, I04, and I04-1 of the Diamond Light Source UK for beam time (proposal mx9948) and assistance with data collection. Finally, we thank Prof. Jirawat Yongsawatdigul (Suranaree University of Technology, Thailand), for control peptides. The work of W.S. was financially supported by Suranaree University of Technology, the Office of the Higher Education Commission under NRU project of Thailand, and the Thailand Research Fund (Grant no. BRG578001, SUT1-102-57-36-18 and SUT1-102-59-12-17). A.A. received a Ph.D. scholarship through the Royal Golden Jubilee Ph.D. Program (Grant no. PHD53K0022). The research leading to these results was conducted as part of the Translocation consortium (www.translocation.eu) and has received support from the Innovative Medicines Initiatives Joint Undertaking under Grant Agreement No. 115525, resources that are composed of financial contributions from the European Union's seventh framework program (FP7/2007-2013) and European Federation of Pharmaceutical Industries and Associations companies in-kind contribution.

\section{Author contributions}

A.A. and M.Z. cloned, expressed, and purified VhChiP. M.Z. crystallized VhChiP and determined crystal structures. A.A. and W.S. performed single-channel electrophysiology experiments. A.S. assisted with low-voltage electrophysiological experiments. A.A. carried out liposome swelling assays. A.K. carried out MD simulations, K.R.P. assisted with the free energy calculations, and U.K. planned and supervised the simulations. A.B. collected crystallography data and maintained the Newcastle Structural Laboratory. B.v.d. B. and W.S. designed research. B.v.d.B. wrote the paper, assisted by W.S. and M.Z.

\section{Additional information}

Supplementary Information accompanies this paper at https://doi.org/10.1038/s41467017-02523-y.

Competing interests: The authors declare no competing financial interests.

Reprints and permission information is available online at http://npg.nature.com/ reprintsandpermissions/

Publisher's note: Springer Nature remains neutral with regard to jurisdictional claims in published maps and institutional affiliations.

(c) (i) Open Access This article is licensed under a Creative Commons Attribution 4.0 International License, which permits use, sharing, adaptation, distribution and reproduction in any medium or format, as long as you give appropriate credit to the original author(s) and the source, provide a link to the Creative Commons license, and indicate if changes were made. The images or other third party material in this article are included in the article's Creative Commons license, unless indicated otherwise in a credit line to the material. If material is not included in the article's Creative Commons license and your intended use is not permitted by statutory regulation or exceeds the permitted use, you will need to obtain permission directly from the copyright holder. To view a copy of this license, visit http://creativecommons.org/ licenses/by/4.0/.

(C) The Author(s) 2018 